\title{
Pathologic Stage III Esophageal Squamous Cell Carcinoma AJCC v8
}

National Cancer Institute

\section{Source}

National Cancer Institute. Pathologic Stage III Esophageal Squamous Cell Carcinoma

A/CC v8. NCI Thesaurus. Code C133536.

Stage III includes: IIIA: (T1, N2, M0, Any G, Tumor location: Any); (T2, N1, M0, Any G, Tumor location: Any); IIIB: (T2, N2, M0, Any G, T umor location: Any); (T3, N1-2, M0, Any G, Tumor location: Any); (T4a, N0-1, M0, Any G, Tumor location: Any). T1: T umor invades the lamina propria, muscularis mucosae, or submucosa. T2: Tumor invades the muscularis propria. T3: Tumor invades adventitia. T4a: Tumor invades the pleura, pericardium, azygos vein, diaphragm, or peritoneum. N0: No regional lymph node metastasis. N1: Tumor with metastasis in one or two regional lymph nodes. N2: Tumor with metastasis in three to six regional lymph nodes. M0: No distant metastasis. (AJCC 8th ed.) 